\title{
PROPUESTA ACTIVIDADES Y ESTRATEGIAS PEDAGOGICAS QUE PROMUEVAN UNA CULTURA DE PAZ Y CAPACIDAD DE RESOLUCION DE CONFLICTOS EN INSTITUCIONES EDUCATIVAS VENEZOLANAS.
}

María Alejandra Aguilar ${ }^{\mathbf{1}}$ : Universidad Metropolitana. Venezuela maguilar@unimet.edu.ve

Natalia Castañón²: Universidad Metropolitana. Venezuela ncastanon@unimet.edu.ve

\section{RESUMEN}

El objetivo de la presente investigación es proponer actividades pedagógicas orientadas a una educación para la paz y resolución de conflictos en escuelas públicas dirigidas a nivel de educación media. El diseño de la investigación es no experimental y de tipo descriptiva. La población son todos aquellos docentes que trabajan en Educación Media en el Municipio Sucre. La muestra estuvo conformada por un total de 19 docentes de educación pública, quienes se desempeñan total o parcialmente en educación media general. El instrumento seleccionado para la recolección de datos fue el cuestionario, por permitir la recopilación de datos precisos, provenientes de una manera directa de nuestra unidad de análisis, así como también por sus ventajas específicas como lo son la versatilidad, el ahorro de costo y el tiempo. Se llevó a cabo un análisis cualitativo a partir de los resultados arrojados en el cuestionario. Los resultado indican que son 12 las actividades y estrategias más prácticas, viables y eficientes utilizadas en la práctica educativa para construir una cultura de paz y resolución de conflictos, las cuales son: educar con el ejemplo, hablar de violencia, el

\footnotetext{
${ }^{1}$ Autor correspondiente:

María Alejandra Aguilar: Facultad de Ciencias y Artes de la Universidad Metropolitana. Venezuela Correo:
}

2 Autor correspondiente:

Natalia Castañón: Facultad de Ciencias y Artes de la Universidad Metropolitana. Venezuela

Correo: ncastanon@unimet.edu.ve 
diálogo y la comunicación, incorporar los valores en cada actividad de la rutina, analizar películas (Cine foro), talleres para padres, dinámicas de roles, juegos cooperativos, el teatro, actividades recreativas, poner en práctica la negociación, la mediación, la conciliación y el arbitraje como estrategias alternativas para la resolución de conflictos y manejo preventivo de la violencia.

PALABRAS CLAVES: Violencia escolar, cultura de paz, resolución de conflictos.

\title{
PROPOSED ACTIVITIES AND TEACHING STRATEGIES THAT PROMOTE A CULTURE OF PEACE AND CONFLICT RESOLUTION CAPABILITY IN VENEZUELAN EDUCATIONAL INSTITUTIONS.
}

\begin{abstract}
The main aim of this research is to suggest a group of pedagogical activities directed towards an education for peace and conflict solving in public schools at secondary level. The design of this research is non-experimental and its type is descriptive. The population is made up of all the teachers that work at secondary level at Municipio Sucre. The sample consisted of a total of 19 teachers in public education working parttime or full-time at secondary level. The instrument selected for the collection of data was the questionnaire, not only because it permits the compilation of precise data that come directly from our unit of analysis, but also for its specific advantages such as flexibility and cost and time saving. We conducted a qualitative analysis using the results obtained from the questionnaire. The results indicate that 12 activities and strategies are the most practical, viable and efficient to be used in the educational process in order to build a culture of peace and conflict solving. These activities are: educate by example, talk about violence, dialog and communication, incorporate values in every activity, analyze films (film forum), workshop for parents, role plays, cooperative games, theater, leisure activities, and practice negotiation, mediation, conciliation and arbitration as alternative strategies to conflict solving and the preventive management of violence.
\end{abstract}

KEYWORDS: Scholastic violence, peace culture, conflicto solving. 


\title{
1. INTRODUCCIÓN
}

Según la Declaración sobre una Cultura de Paz de las Naciones Unidas (1999) la paz es un término que no sólo se refiere a la ausencia del conflicto o guerra, sino implica "un proceso positivo, dinámico y participativo en que se promueva el diálogo y se solucionen los conflictos en un espíritu de entendimiento y cooperación mutuos".

Los gobiernos, las organizaciones internacionales y la sociedad civil reconocen la necesidad imperante de eliminar cualquier forma de intolerancia y discriminación en cualquier espacio, sean basadas en la raza, el color, el sexo, el idioma, la religión, la opinión política o de otra índole, el origen nacional, étnico o social, la propiedad, las discapacidades, el nacimiento u otra condición, y esto se logra a través de la promoción y fortalecimiento de una Cultura de Paz, definida por las Naciones Unidas (1999) como la fusión de los valores, actitudes, tradiciones, comportamientos y estilos de vida, de manera que giren en torno a pilares fundamentales como el compromiso con el arreglo pacífico de los conflictos, el respeto a la vida, el fin de la violencia y la promoción y la práctica de la no violencia por medio de la educación, el diálogo y la cooperación, entre otros.

Se puede observar como se está masificando un entusiasmo, especialmente en Venezuela, basado en iniciativas que propicien un entorno nacional que favorezca la paz, con lo cual los beneficios serán innumerables para las personas, los grupos y las naciones del mundo.

\begin{abstract}
La cultura de paz supone ante todo un esfuerzo generalizado para modificar mentalidades y actitudes con ánimo de promover la paz. Significa transformar y prevenir los conflictos que puedan engendrar violencia y restaurar la paz y la confianza en poblaciones que emergen de la guerra. Pero su propósito trasciende lo límites de los conflictos armados para hacerse extensivo también a las escuelas y los lugares de trabajo del mundo entero, los parlamentos y las salas de prensa, las familias y los lugares de recreo. (Unesco S/F).
\end{abstract}

Esta investigación se centra en la cultura de paz en el ámbito educativo, donde distintos entes desempeñan una función clave en la promoción la misma: los padres, los maestros, los políticos, los trabajadores sociales y las organizaciones no gubernamentales. De la misma forma se aborda con detalle la importancia de la educación en todos los niveles como elemento promotor de la Cultura de Paz, como vehículo fundamental para la construcción de la misma, considerando:

1. La promoción de la resolución pacífica de los conflictos, el respeto y entendimiento mutuo.

2. La posibilidad que todas las personas a todos los niveles desarrollen aptitudes para el diálogo, la negociación, la formación de consenso y la solución pacífica de controversias.

Por esto, debe entenderse la cultura de paz como la acción que niños y adultos rechacen de forma colectiva los hechos violentos, y mucho más allá, que comprendan, 
respeten y exijan valores fundamentales como la libertad, la justicia, la democracia, los derechos humanos, la tolerancia, la igualdad y la solidaridad, actuando con empatía.

\section{METODOLOGÍA}

2.1. Objetivo de la investigación

Proponer actividades pedagógicas orientadas a una educación para la paz y resolución de conflictos en escuelas públicas dirigidas a nivel de educación media.

\subsection{Diseño de la investigación}

La investigación es de diseño No Experimental. Según Hernández, Fernández y Baptista (2006), este tipo de diseño "es la relación que se ejecuta sin manipular deliberadamente variables, se trata de estudios donde se hace variar en forma intencional las variables independientes para ver su efecto sobre otras variables".

\subsection{Tipo de investigación}

Esta investigación es de tipo descriptiva ya que pretende referir las actividades pedagógicas sugeridas por los docentes que sean más viables en la práctica educativa

\subsection{Población y muestra}

La población son todos aquellos docentes que trabajan en Educación Media en el Municipio Sucre. La muestra estuvo conformada por un total de 19 docentes de educación pública, quienes se desempeñan total o parcialmente en educación media general.

\subsection{Técnicas e instrumentos de recolección de datos}

El instrumento seleccionado para la recolección de datos fue el cuestionario, por permitir la recopilación de datos precisos, provenientes de una manera directa de nuestra unidad de análisis, así como también por sus ventajas específicas como lo son la versatilidad, el ahorro de costo y el tiempo.

2.6. Técnicas de recolección de datos 
Se llevó a cabo un análisis cualitativo a partir de los resultados arrojados en el cuestionario.

\section{ANÁLISIS Y DISCUSIÓN}

\subsection{Violencia en Venezuela.}

Según el observatorio venezolano de violencia (OVV, 2013), en el año 2013 se observó un incremento en los homicidios, "los cuales continuaron con un crecimiento en las grandes ciudades que ha acompañado el cambio poblacional de las urbes". Las muertes violentas representan en Venezuela el $12 \%$ de la mortalidad general. El impacto económico y social de esta situación es relevante, pues los decesos ocurren de forma mayoritaria entre los hombres jóvenes, quienes se encuentran en edad productiva, por lo cual se afecta la composición de la pirámide poblacional, las relaciones de dependencia demográfica y está disminuyendo la esperanza de vida de los hombres.

Venezuela continúa estando entre los cinco países más violentos del mundo (junto con Honduras, El Salvador, Costa de Marfil y Jamaica), el incremento ocurrido evidencia una desaceleración en las muertes violentas, quizá como resultado de la mayor conciencia de la población, de las tendencias inhibitorias en el comportamiento de los ciudadanos y en las políticas de seguridad implementadas por los gobiernos nacional y locales.

Venezuela necesita ser pacificada y la manera de empezar es desde los centros educativos, niños de temprana edad y maestros formados en el área de educación para la paz y resolución de conflictos es la principal estrategia para combatir este lamentable mal.

\subsection{Violencia en los centros educativos.}

La escalada de violencia en los centros educativos en los últimos años es un hecho que afecta y preocupa a todos como sociedad. Sin distinción de clase social ni ubicación geográfica las escuelas se desbordan de hechos violentos que interrumpen el desarrollo del currículo escolar, la estabilidad de los estudiantes y de los docentes en general, por eso es importante que se eduque para el conflicto. Según Vinyamata (2005) "se debe estar preparado para evitar y superar la guerra, al mismo tiempo que se aprende a luchar sin odio y violencia", expresión vigente y absolutamente necesaria ante el contexto de violencia que viven las instituciones educativas en el país causadas por la radicalización política, la falta de diálogo, distorsión de los valores y carencia de moral. 
Este conjunto debe ser visto desde una perspectiva global, no como un hecho aislado, sino como sucesos que tienen múltiples repercusiones e involucrados directos e indirectos, en la cual no necesariamente se necesita de la fuerza física para dominar o destruir. Si bien las situaciones de conflictos y los problemas son parte de la vida, se debe educar para vivir de forma serena, sensata y superar las dificultades de manera no violenta.

La violencia es un ciclo vicioso, un hecho violento produce rencor y odio, los cuales a su vez producen más violencia. Por esto es importante tener en cuenta las causas de las actitudes y comportamientos que se evidencian en estos hechos, que en muchas ocasiones se relacionan con frustraciones e injusticias. Si conocemos la razón de ser de la violencia, las estrategias de mediación y conciliación podrán ser efectivas, llevándonos hacia una educación que transita por los caminos de paz (Vinyamata, 2005).

Es por ello que abordar la formación docente en este tema es clave y muy especialmente tener en cuenta actividades prácticas que puedan aplicar los docentes en el marco de una cultura de paz y resolución del conflicto.

\section{RESULTADOS}

A continuación se presentan los resultados arrojados en los resultados, tras realizarse un análisis cualitativo de los mismos. Los resultado indican que son 12 las actividades y estrategias más prácticas, viables y eficientes utilizadas en la práctica educativa para construir una cultura de paz y resolución de conflictos, las cuales son:

1) Educar con el ejemplo: la mayoría de la muestra refirió que educar con el ejemplo es una de las estrategias más importantes y efectivas para la construcción de una cultura de paz, la cual deberían poner en práctica todos los docentes, padres, empleados de organismos gubernamentales, etc., ya que la tendencia ha sido "hacer lo que yo digo y no lo que yo hago"; y por el sólo hecho de ser adultos, se es una figura modelo, referente de los niños y adolescentes que están alrededor, sin necesidad de que se tenga nexo alguno. Simplemente el adulto, más aun siendo docentes o padres, debe tener un mensaje coherente, cónsono con su comportamiento. Sin embargo, para lograr esto, la muestra también reporta que se debe pasar por un proceso educativo, de formación y actualización importante en tema de valores y educación para la paz, de manera de poder practicar lo que se pretende fomentar.

2) Hablar de violencia: esta estrategia resultó ser una de las populares en la muestra. Normalmente no se está preparado para afrontar la violencia, los docentes de la muestra manifestaron no saber manejarla, no tener las herramientas para abordarla desde una perspectiva positiva, pacífica. Incluso, es las aulas es poco lo que se habla de la violencia y mucho menos se señala al violento por temor a represalias, a consecuencias, ya que la violencia ha ido incrementando y expresan sorprenderse de lo novedosos que pueden ser los 
padres y estudiantes cuando de violencia se trata, razón por la cual la muestra otorga especial importancia a hablar del tema, sin miedo, sin naturalidad (porque no es algo natural) pero sin violencia, abordando el tema con los estudiantes, tratando de generar reflexiones en función de los valores.

3) El diálogo y la comunicación: los docentes reportaron que haciendo uso del diálogo han podido tener resultados positivos en el tema de violencia. La muestra manifiesta que cuando se inicia un proceso de diálogo, que generalmente en este caso va acompañado de una suerte de mediación por parte del docente, los estudiantes tienden a calmarse al exponer sus diferentes puntos de vista, al tiempo que se permiten escuchar la posición de la otra parte y encontrar la raíz o causa del conflicto, de manera de poder actuar en consecuencia. Los docentes de la muestra que han practicado con mayor frecuencia esta estrategia en la resolución de conflictos, reportaron que han tenido estudiantes que para resolver sus problemas ya no necesitan al mediador, sino que lo utilizan como estrategia permanente antes de recurrir a la violencia.

4) Incorporar los valores en cada actividad de la rutina: Toda la muestra expresó su preocupación por ante el tema de los valores, ya que consideran que la causa de mayor magnitud que está generando la violencia en este país es que los valores se han desvirtuado. Los docentes señalan que vale más un teléfono celular que la vida de una persona; que por dinero hay quienes hacen lo que sea, inclusive matar a sus familiares. Con este escenario, los docentes de esta muestra han decidido incorporar los valores, de manera formal, y trabajarlos en cada actividad y en cada momento de la rutina escolar y extra curricular. Indican que el robo intencionado de algún material u objeto ajeno se percibe desde edades tempranas, por lo que cada vez que sucede un evento de esta naturaleza, discuten el tema en grupo, de manera individual, exponen la situación a la reflexión y resaltan los valores positivos. En este sentido, esta estrategia les ha resultado de gran ayuda para minimizar la violencia en el aula y en la escuela.

5) Analizar películas (Cine foro): Existen cantidades de películas interesantes, de gran calidad, basadas en hechos de la vida real, que tocan muy de cerca el escenario de la violencia y cómo ha sido abordado y resuelto. Esta estrategia fue reportada por la muestra como la más emotiva y que remueve sentimientos muy internos, ya que a través de una película, intencionalmente seleccionada, ayuda a los estudiantes a ubicarse, a ponerse en los zapatos del otro, a entender la realidad que vive él o su compañero y, lo más importante, a buscar opciones para salir de esa realidad. Además es una estrategia que los estudiantes disfrutan a cabalidad y que los docentes aprovechan para ilustrar un panorama que se pueda estar presentando.

6) Talleres para padres: definitivamente abordar la violencia debe ser un trabajo conjunto, tal como lo mencionan los docentes de la muestra, quienes consideran que otra de las causas de la misma es la familia. En este sentido, las escuelas donde laboran estos docentes han tomado la iniciativa de hacer 
talleres para padres con temas relacionados con la violencia. Ellos manifiestan que la receptividad al inicio es muy baja, eran pocos los padres que respondían e iban. Sin embargo, tras un proceso de sensibilización y de acercamiento de la escuela con el hogar, se ha ido ganando terreno y cada vez son más los padres que asisten. Resaltan la importancia de hablarles en un idioma que entiendan, que se les haga cercano y conocido lo que están oyendo, y que también sea una oportunidad para reflexionar y generar cambios importantes, por el bien de sus hijos.

7) Dinámicas de roles: esta estrategia ha sido de gran efectividad reportada por la muestra, ya que los docentes, conocedores de su grupo y entorno, la han llevado a cabo con frecuencia. Esta consiste en repartir un rol o un papel que a veces no son los aceptados a determinados estudiantes, los cuales desempeñan el papel de acuerdo a sus conocimientos, educación y experiencia. En este sentido, los docentes de la muestra han manifestado innumerables situaciones que han generado reflexión en sus estudiantes y que han desembocado en un cambio profundo, importante en su manera de comportarse y de ver la vida.

8) Juegos cooperativos: el juego es una actividad que está presente, de una u otra forma, en todo ser humano y aunque habitualmente se relaciona con la infancia, también se manifiesta a lo largo de la vida. La muestra otorgó gran importancia a este tipo de actividades por la importancia del juego en el aprendizaje humano. De hecho, los docentes, influidos por la teoría cognoscitiva de Piaget, hacen de sus aulas un lugar activo, en donde la curiosidad de los niños, en el caso de la educación preescolar, sea satisfecha con materiales adecuados para descubrir, explorar, discutir, debatir. Del mismo modo, el juego, de cualquier índole, permite determinar la evolución del concepto de juicio moral en niños y jóvenes, lo cual lo hace necesario y atractivo para disminuir la violencia. El tema de que sea cooperativo, con una buena mediación docente, invita a los estudiantes a ponerse en el lugar del otro, a ser empáticos y reflexionar acerca de las diferentes conductas o posturas ante el conflicto.

9) El teatro: los docentes de la muestra reportaron que a través de esta estrategia se produce un proceso de expresión y comunicación que acerca a los participantes a los usos y aplicaciones de la práctica teatral y artística para lograr la disminución de la violencia, el manejo de los conflictos y la creación de una cultura de paz, al tiempo que forma parte de los planes de estudio de varios niveles de Educación Media. Igualmente, reportan que esta estrategia ayuda a los estudiantes a canalizar sus emociones, a concentrarse e identificar la causa de la violencia y abordarla positivamente.

10)Actividades recreativas: la mayoría de la muestra mencionaron este tipo de actividades como propiciadoras de la paz, argumentando que la convivencia puede llevarse a cabo, de manera más sencilla, motivadora, a través de estas actividades, especialmente cuando se trata un tema tan complejo como es abordar la violencia. La muestra manifestó que a través de estas actividades los estudiantes tienden a desahogarse, calmarse, se sienten parte de un grupo, y 
Violencia escolar - cultura de paz - resolución de conflictos.

pasan un momento agradable, están activos y contentos en vez de pelear. Además, este tipo de actividades permiten que los docentes se acerquen a los estudiantes desde otra perspectiva.

11)Poner en práctica la negociación, la mediación, la conciliación y el arbitraje como estrategias alternativas para la resolución de conflictos: Estos términos inicialmente resultaban muy lejanos para la muestra, debido al desconocimiento. Sin embargo, a medida que aplicaban la negociación, en donde ambas partes sintieron que ganaron; la mediación permanente, la conciliación para evitar llegar a la violencia y, por qué no, al arbitraje cuando las partes no logran ponerse de acuerdo y llegar a una resolución ganar ganar; se fueron haciendo más familiares, hasta el punto que los estudiantes de los docentes de la muestra pueden llegar a utilizarlas sin necesidad de un mandato o sugerencia suprema.

12)Manejo preventivo de la violencia: Esta última estrategia, pero no menos importante, fue considerada por toda la muestra como un elemento que requiere ser concientizado, sensibilizado, interiorizado, comprendido y ejecutado. En la medida en que se lleven a cabo estrategias y actividades para abordar la violencia, se les está enseñando a los estudiantes como resolver los conflictos, que son inherentes al ser humano, de manera no violenta, lo cual, si duda alguna, está generando un proceso preventivo de la violencia a futuro. Los docentes de la muestra manifestaron que la educación es una de las pocas estrategias preventivas de la violencia, ya que conlleva cambios profundos en una cultura que, bien planificada y encaminada, puede llevar a una verdadera paz; no como ausencia de guerra, sino como un estado de bienestar, de reducida violencia y elevada justicia, donde el diálogo y el entendimiento mutuo son los pilares fundamentales para la resolución de cualquier conflicto.

En este sentido, haciendo referencia al último punto planteado, es importante detenerse para mencionar y confirmar que, definitivamente, la educación es un factor primordial para la disminución de los niveles de violencia, así lo consideran, de manera unánime los 19 docentes de la muestra y las investigadoras de este estudio. Y resulta de gran utilidad hacer énfasis en un comentario escrito por uno de los docentes de la muestra, quien expresó lo siguiente:

"Si es importante la educación para disminuir el índice de violencia. Si se asume la educación como un proceso dinámico que busca como objetivo formar al ser humano de forma integral, desde lo cognitivo hasta lo afectivo y social. Entonces es a través de la educación que podemos educarnos para convivir con el otro, para resolver conflictos sin utilizar la violencia. En fin, es a través de la educación que podemos conocer, entender $e$ interiorizar que hay otras formas de convivir; en paz, en un ambiente de justicia, solidaridad con el otro."

Finalmente, como docentes, es importante que se tenga la firme convicción y sobre todo la claridad en la importancia que posee el proceso educativo en todos los 
aspectos de la vida del ser humano, no solo para disminuir la violencia, sino para transformar sociedades, culturas y alcanzar la paz.

\section{CONCLUSIONES}

A través del desarrollo de este trabajo de investigación se propuso una serie de actividades orientadas a desarrollar una educación para la paz y la resolución no violenta de conflictos en escuelas públicas de educación media y que fueron aplicadas por los docentes de la muestra, obteniendo así un listado de 12 actividades y estrategias más prácticas, viables y eficientes para construir una cultura de paz.

El objetivo de la investigación fue alcanzado satisfactoriamente, ya que se planteó brindar a los docentes participantes actividades y estrategias pedagógicas que pueda aplicar en la escuela y en el aula, orientadas a lograr la convivencia pacífica y así una cultura de paz.

La investigación y el acercamiento con los docentes de la muestra permitió el análisis y la reflexión sobre la realidad del entorno escolar inmediato que viven los participantes, la cual es compleja y conflictiva; a la vez que se ofrecieron una serie de estrategias para que el docente pueda actuar en consecuencia bajo los principios de la paz positiva. En este sentido, los resultados de la investigación parecen haber concedido una dosis de motivación que le permitió a los docentes participantes identificar nuevos y diferentes elementos que llevados con su esfuerzo y dedicación se orientarán a la consolidación del tránsito hacia una cultura de paz.

El rol del docente actualmente lo ha convertido, inexorablemente, en un agente clave en el compromiso de disminuir la violencia y caminar hacia una cultura de paz. El docente, como formador de seres humanos tiene la responsabilidad de siempre dar lo mejor de sí y procurar así la educación integral de los individuos. A pesar de la adversa realidad en este país -quizá en el mundo entero- los valores del docente deben prevalecer y hacerse notar en su labor diaria, si en la familia estos valores se han perdido, como afirman muchos de los participantes, la escuela pasa a ser el espacio para justamente reforzar los valores desvirtuados en otros espacios, por ello el esfuerzo y el trabajo en equipo deben ser constantes para lograr la paz, como ideal de convivencia entre los seres humanos. La educación es, sin duda alguna, un factor esencial en la consolidación de una cultura de paz en la sociedad.

Finalmente y en términos generales, al observar los resultados de la investigación se puede concluir que se generó un impacto significativo en la muestra que participó de este estudio. En principio, hubo un impacto en los conocimientos de los docentes, también en la adquisición de las estrategias y herramientas para 
disminuir la violencia escolar, ya que según lo expresado por ellos, disminuyó la cantidad de veces que se presentó la violencia en el aula, aunque sin extinguirla, gracias a la implementación de estrategias aprendidas.

\section{BIBLIOGRAFIA}

HERNÁNDEZ, R., FERNÁNDEZ, C. Y BAPTISTA, P. (2006). Metodología de la Investigación. México: Mc Graw Hill/Interamericana Editores S.A., 3ra. Edición.

NACIONES UNIDAS (1999). Declaración y Programa de Acción sobre una Cultura de Paz. Documento en línea: http://www.unesco.org/cpp/sp/proyectos/suncofp.pdf. Fecha de consulta: [13/03/14]

OBSERVATORIO VENEZOLANO DE VIOLENCIA (2013). Violencia, Ciudadanía y miedo en Caracas. En línea: http://observatoriodeviolencia.org.ve/ws/. Fecha de consulta: $[12 / 03 / 14]$

UNESCO (s.f). Mensaje del Director General de la UNESCO con Motivo del Año Internacional de la Cultura de la Paz. En línea: http://www.unesco.org/cpp/sp/proyectos/dgmessagesp.htm. Fecha de consulta: [18/02/04]

VINYAMATA, E. (2005). Conflictología: curso de resolución de conflictos. España: Editorial Ariel. 2da edición.

\section{Natalia Castañón:}

Educación superior: Lic. en Educación (Universidad Metropolitana,1991), Maestría en Psicología Escolar (Universidad Complutense de Madrid, 1993), Doctorado en Filosofía y Ciencias de la Educación (Universidad Complutense de Madrid, 1999).

Responsabilidades Académicas: Profesora a tiempo completo de la Universidad Metropolitana (1993-), jefe del departamento de didáctica (2002-), jefe del departamento de humanidades (2009-), profesor investigador.

Productos Académicos: Tutora de 29 tesis de pre y postgrado. Publicaciones en once revistas o libros arbitrados a nivel nacional e internacional. Participación en 30 Congresos Nacionales e Internacionales de ponencias arbitradas. Asesora y/o consultora de 22 proyectos educativos nacionales e internacionales (Banco Mundial, IBM, PDVSA, Dividendo Voluntario para la Comunidad, Procter\&Gamble, Sanitarios Maracay, Universidad Metropolitana, Fundación Cisneros, Alcaldía de Chacao, entre otros). Líder de 5 proyectos de investigación nacionales e internacionales (Conicit, Programa Alfa de la Comunidad Europea, Universidad Metropolitana). 
Proyectos profesionales: trabajar en la educación superior, específicamente dos áreas: formación de estudiantes e investigación. Son varios ejes de interés: emprendeduría (plan estratégico incubación de empresas y solución a problemas sociales), cultura de paz (educación al desarme, educación para la democracia, educación derechos humanos, etc), redes sociales (tecnología y educación) y análisis del contexto (literatura, arte, filosofía, historia y pedagogía).

María Alejandra Aguilar:

Educación superior: Lic. en Educación (Universidad Metropolitana,1998), Especialización en Informática Educativa (Universidad Simón Bolívar, 2004), Especialización en Entornos Virtuales de Aprendizaje (VirtualEduca, 2010).

Responsabilidades Académicas: Profesora a tiempo completo de la Universidad Metropolitana (2001-2005), Profesora a tiempo parcial (2010 - ).

Productos Académicos: Tutora de 21 tesis de pre y postgrado. Publicaciones en dos revistas a nivel nacional. Participación en 4 congresos nacionales e internacionales de ponencias arbitradas. Coordinadora y asistente de proyectos educativos nacionales (Dividendo Voluntario para la Comunidad, Universidad Metropolitana, Fundación Cisneros, Alcaldía de Chacao, independientes).

Proyectos profesionales: el trabajo en educación superior orientada a la formación de estudiantes y a la investigación en los temas de educación para la paz y resolución de conflictos sobre la base de los derechos humanos, de la justicia y la democracia, de la interculturalidad, entre otros), el impacto de la tecnología en la educación, el emprendimiento como forma de desarrollo sostenible, y la capacitación, actualización y acompañamiento efectivo de docentes y futuras docentes en su práctica pedagógica. 